\title{
Existence and construction of a quantum channel with given inputs and outputs
}

\author{
GUO ZhiHua \& CAO HuaiXin* \\ College of Mathematics and Information Science, Shaanxi Normal University, Xi'an 710062, China
}

Received June 16, 2012; accepted September 17, 2012

\begin{abstract}
We derive that sufficient and necessary conditions for existence of a quantum channel $\phi$ and a generalized unitary operation $\mathcal{E}$ sending $A_{i}$ to $B_{i}(1 \leqslant i \leqslant k)$ for two given families $\left\{A_{i}\right\}_{i=1}^{k},\left\{B_{i}\right\}_{i=1}^{k}$ of matrices, respectively. As an application, a sufficient and necessary condition for existence of a unitary duality quantum computer with given input-output states is obtained.
\end{abstract}

existence, quantum channel, generalized unitary operation, duality quantum computer

Citation: Guo Z H, Cao H X. Existence and construction of a quantum channel with given inputs and outputs. Chin Sci Bull, 2012, 57: 4346-4350, doi: 10.1007/s11434012-5506-3

\section{Introduction}

The theory of quantum information and quantum computation [1] is a result of the effort to generalize classical information theory to the quantum world. According to quantum mechanics, a quantum system is described by a finite dimensional Hilbert space $\mathcal{H}$, a state of the system is described by a unit vector in $\mathcal{H}$, or a density operator (a positive operator of trace 1). An evolution of the system is called a quantum operation and denoted by a unitary operator (called a quantum gate) or a completely positive trace-preserving (CPTP) mapping (called a quantum channel). A convex combination of unitary operators is called a generalized quantum gate [2-4], which came from a duality quantum computer [5-14].

In quantum information science, it is necessary and useful to discuss the existence and construction of a quantum channel that maps a given family of density operators to another.

We consider a composite quantum system $\mathcal{H}_{A} \otimes \mathcal{H}_{B}$ composed of two parts, finite dimensional Hilbert spaces $\mathcal{H}_{A}$ and $\mathcal{H}_{B}$. The first example is the following.

Quantum cloning question (QCQ). Given a set $\left\{\rho_{1}, \rho_{2}, \ldots, \rho_{k}\right\}$ of states on $\mathcal{H}_{A}$ and a state $\Sigma$ on $\mathcal{H}_{B}$, to find a quantum channel $\phi: B\left(\mathcal{H}_{A}\right) \rightarrow B\left(\mathcal{H}_{B}\right)$ such that

*Corresponding author (email: caohx@ snnu.edu.cn)

$$
\phi\left(\rho_{s} \otimes \Sigma\right)=\rho_{s} \otimes \rho_{s}(s=1, \ldots, k),
$$

where $B(\mathcal{H})$ denotes the algebra of all bounded linear operators on $\mathcal{H}$. If such a $\phi$ exists, we call $\rho_{1}, \rho_{2}, \ldots, \rho_{k}$ can be cloned simultaneously $[15,16]$.

The second example is the so-called classical correlation question.

Classical correlation question (CCQ). Given a state $\rho$ on $\mathcal{H}_{A} \otimes \mathcal{H}_{B}$, to find a von Neumann measurement $\Pi=$ $\left\{\Pi_{s}^{A} \otimes \Pi_{t}^{B}: 1 \leqslant s \leqslant m, 1 \leqslant t \leqslant n\right\}$ such that

$$
\Pi(\rho):=\sum_{s=1}^{m} \sum_{t=1}^{n}\left(\Pi_{s}^{A} \otimes \Pi_{t}^{B}\right) \rho\left(\Pi_{s}^{A} \otimes \Pi_{t}^{B}\right)=\rho,
$$

where $\Pi_{s}^{A}$ and $\Pi_{t}^{B}$ are one-dimensional orthogonal projections on $\mathcal{H}_{A}$ and $\mathcal{H}_{B}$, respectively. If such a $\Pi$ exists, we call the state $\rho$ is classical correlated [17-22]. Gisin [23] presented a theorem in 1991 that states that any pure entangled two-qubit state violates the $\mathrm{CHSH}$ inequality. Specifically, the $\mathrm{CHSH}$ inequality is both sufficient and necessary for the separability of two-qubit states and any pure entangled quantum state violates one of Bell-like inequalities [24].

Thus, the following question arises naturally: given families $\left\{A_{i}\right\}_{i=1}^{k} \subset \mathcal{M}_{N}$ and $\left\{B_{i}\right\}_{i=1}^{k} \subset \mathcal{M}_{M}$ of matrices, to discuss the existence and construction of a quantum channel 
$\phi: \mathcal{M}_{N} \rightarrow \mathcal{M}_{M}$ such that $\phi\left(A_{i}\right)=B_{i}$ for every $1 \leqslant i \leqslant k$ $\mathcal{M}_{S, T}$ denotes the set of all $S \times T$ matrices and when $S=T$, write it as $\mathcal{M}_{S}$.

In Section 2, some preliminaries will be given. In Section 3 , we will derive that sufficient and necessary conditions for existence of a quantum channel $\phi$ and a generalized unitary operation $\mathcal{E}$ sending $A_{i}$ to $B_{i}(1 \leqslant i \leqslant k)$ for two given families $\left\{A_{i}\right\}_{i=1}^{k},\left\{B_{i}\right\}_{i=1}^{k}$ of matrices, respectively. In Section 4, a sufficient and necessary condition for existence of a unitary duality quantum computer with given input-output states will be obtained as an application.

\section{Preliminaries}

For a complex matrix $A, A^{\dagger}$ denotes the transpose of the complex conjugate of $A$. We say a square matrix $A$ is Hermitian if $A=A^{\dagger}, A$ is positive semi-definite if $A$ is Hermitian and its eigenvalues are nonnegative. $\mathcal{M}_{N}\left(\mathcal{M}_{M}\right)=\mathcal{M}_{N} \otimes \mathcal{M}_{M}$ is the collection of all $N \times N$ block matrices with $M \times M$ matrices as entries. A linear map $\phi: \mathcal{M}_{N} \rightarrow \mathcal{M}_{M}$ is positive if $\phi(A)$ is positive semi-definite for any positive semi-definite matrix $A$ in $\mathcal{M}_{N}$. Define $I_{p} \otimes \phi: \mathcal{M}_{p}\left(\mathcal{M}_{N}\right) \rightarrow \mathcal{M}_{p}\left(\mathcal{M}_{M}\right)$ by $\left(I_{p} \otimes \phi\right)\left(\left[A_{j, k}\right]\right)=\left[\phi\left(A_{j, k}\right)\right]$. Then we call $\phi$ is completely positive (CP) if $I_{p} \otimes \phi$ is positive for all positive integers $p$.

Consider an $N \times M$ rectangular matrix $A=\left[a_{i j}\right]$, we define the reshaping of $A$ as

$$
\operatorname{res}(A)=\left(a_{11}, \ldots, a_{1 M}, a_{21}, \ldots, a_{2 M}, \ldots, a_{N 1}, \ldots, a_{N M}\right)^{t},
$$

where $X^{t}$ denotes the transpose of a matrix $X$. Clearly, the length of $\operatorname{res}(A)$ is $N M$.

Conversely, any vector of length $N M$ may be reshaped into an $N \times M$ rectangular matrix. For example,

$$
\left(a_{1}, a_{2}, a_{3}, a_{4}\right)^{t} \leftrightarrow\left(\begin{array}{ll}
a_{1} & a_{2} \\
a_{3} & a_{4}
\end{array}\right) .
$$

For arbitrary $M N \times S T$ matrix $C$, usually we use both indices to denote $C=\left[a_{i j}\right]$, but more conveniently, we will use the block-form of $C$ as

$$
C=\left[C_{m, n}\right] \in \mathcal{M}_{M, N}\left(\mathcal{M}_{S, T}\right)
$$

and then write as

$$
C=\left[C_{m, n}\right]=\left[c_{n, \mu}^{m, \mu}\right],
$$

where $c_{n, \nu}^{m, \mu}$ denotes the $(\mu, v)$-entry of $(m, n)$-block matrix $C_{m, n}$. For example,

$$
C=\left(\begin{array}{rr|rr}
c_{1,1} & c_{1,1} & c_{1,1} & c_{1,1} \\
1,1 & 1,2 & 2,1 & 2,2 \\
c_{1,2} & c_{1,2} & c_{1,2} & c_{1,2} \\
1,1 & 1,2 & 2,1 & 2,2 \\
\hline c_{2,1} & c_{2,1} & c_{2,1} & c_{2,1} \\
1,1 & 1,2 & 2,1 & 2,2 \\
c_{2,2} & c_{2,2} & c_{2,2} & c_{2,2} \\
1,1 & 1,2 & 2,1 & 2,2
\end{array}\right)=\left[c_{n, \nu}^{m, \mu}\right] .
$$

Especially, when $C=A \otimes B$, where

$$
A=\left[a_{m n}\right] \in \mathcal{M}_{M, N}, B=\left[b_{\mu \nu}\right] \in \mathcal{M}_{S, T},
$$

we have $c_{n, v}^{m, \mu}=a_{m n} b_{\mu v}$.

For arbitrary $M N \times S T$ matrix $C$ defined by eq. (2.2), we define a block-matrix

$$
C^{K}=\left[C_{m, \mu}^{K}\right]=\left[c_{\mu, \nu}^{m, n}\right],
$$

where $c_{\mu, \nu}^{m, n}$ denotes the $(n, v)$-entry of $(m, \mu)$-block matrix $C_{m, \mu}^{\kappa}$.

Let $\phi: \mathcal{M}_{N} \rightarrow \mathcal{M}_{M}$ be a completely positive map. Then from Choi's Theorem [25], we know that $\phi$ can be represented as

$$
\phi(\rho)=\sum_{i=1}^{k} V_{i} \rho V_{i}^{\dagger}, \quad \forall \rho \in \mathcal{M}_{N} .
$$

Now, we reshape $V_{i}$ into a length $M N$ column vector $\operatorname{res}\left(V_{i}\right)$ following eq. (2.1). Define

$$
V_{\phi}:=\left(\operatorname{res}\left(V_{1}\right), \operatorname{res}\left(V_{2}\right), \ldots, \operatorname{res}\left(V_{k}\right)\right),
$$

and put

$$
D_{\phi}:=V_{\phi} V_{\phi}^{\dagger}=\sum_{i=1}^{k}\left(\operatorname{res}\left(V_{i}\right)\right)\left(\operatorname{res}\left(V_{i}\right)\right)^{\dagger},
$$

which is an $M N \times M N$ matrix.

The following properties on matrix $D_{\phi}$ were essentially derived in [26].

Proposition 2.1 Suppose that $\phi: \mathcal{M}_{N} \rightarrow \mathcal{M}_{M}$ is a completely positive map, then

(1) the matrix $D_{\phi}$ defined by eq. (2.5) is uniquely determined by $\phi$ and positive semi-definite; moreover, $D_{a \phi_{1}+b \phi_{2}}=$ $a D_{\phi_{1}}+b D_{\phi_{2}}$ holds for arbitrary positive numbers $a, b$ and $\mathrm{CP}$ maps $\phi_{1}$ and $\phi_{2}$.

(2) $\phi$ is unital if and only if $\operatorname{tr}_{2} D_{\phi}:=\sum_{\mu=1}^{N} z_{n, \mu}^{m, \mu}=I_{M}$; and $\phi$ is trace-preserving if and only if $\operatorname{tr}_{1} D_{\phi}:=\sum_{m=1}^{M} z_{m, \mu}^{m, v}=I_{N}$, where $\operatorname{tr}_{S} X$ denotes that the partial trace of $X$ for the $s$ th system and $I_{M}$ the $M \times M$ identity matrix.

(3) $\operatorname{res}(\phi(\rho))=D_{\phi}^{K} \operatorname{res}(\rho)$ for each $\rho \in \mathcal{M}_{N}$.

\section{Main results}

Theorem 3.1 Let $\left\{A_{i}\right\}_{i=1}^{k} \subset \mathcal{M}_{N}$ and $\left\{B_{i}\right\}_{i=1}^{k} \subset \mathcal{M}_{M}$. Then the following conditions are equivalent.

(a) There exists a quantum channel $\phi: \mathcal{M}_{N} \rightarrow \mathcal{M}_{M}$ such that $\phi\left(A_{i}\right)=B_{i}$ for every $1 \leqslant i \leqslant k$.

(b) There exists an $M N \times M N$ positive semi-definite matrix $E$ such that $\operatorname{tr}_{1} E=I_{N}$ and

$$
\operatorname{res}\left(B_{i}\right)=E^{\kappa} \operatorname{res}\left(A_{i}\right)(1 \leqslant i \leqslant k) .
$$

Proof $\quad(a) \Rightarrow(b)$ Suppose that there exists a quantum channel $\phi$ satisfying $\phi\left(A_{i}\right)=B_{i}$ for every $1 \leqslant i \leqslant k$. Take 
$E=D_{\phi}$. Proposition 2.1 implies that $E$ is an $M N \times M N$ positive semi-definite matrix with $\operatorname{tr}_{1} E=I_{N}$ and satisfies (3.1).

(b) $\Rightarrow$ (a) Suppose there exists an $M N \times M N$ positive semidefinite matrix $E$ satisfying $\operatorname{tr}_{1} E=I_{N}$ and (3.1). First, $E$ has a decomposition $E=F F^{\dagger}$ for some $M N \times M N$ matrix $F=\left[F_{1}, F_{2}, \ldots, F_{M N}\right]$. Furthermore, we reshape the jth column $F_{j}$ of $F$ into an $M \times N$ matrix $V_{j}$ for every $1 \leqslant j \leqslant M N$. Thus, $\operatorname{res}\left(V_{j}\right)=F_{j}$ for all $1 \leqslant j \leqslant k$. Define $\phi(\rho)=\sum_{j=1}^{M N} V_{j} \rho V_{j}^{\dagger}$. Then Choi's Theorem reads $\phi: \mathcal{M}_{N} \rightarrow \mathcal{M}_{M}$ is a completely positive map. By (3.1) and Proposition 2.1(3), we get

$$
\operatorname{res}\left(B_{i}\right)=E^{K} \operatorname{res}\left(A_{i}\right)=\operatorname{res}\left(\phi\left(A_{i}\right)\right)
$$

for all $1 \leqslant i \leqslant k$. Lastly, by Proposition 2.1 (2) implies that $\phi$ is trace-preserving and then a quantum channel.

Example 1 Let

$$
\begin{aligned}
& A_{1}=\left(\begin{array}{ll}
1 & 0 \\
0 & 0
\end{array}\right), A_{2}=\left(\begin{array}{ll}
0 & 1 \\
0 & 0
\end{array}\right), \\
& A_{3}=\left(\begin{array}{ll}
1 & 1 \\
2 & 0
\end{array}\right), A_{4}=\left(\begin{array}{ll}
0 & 0 \\
0 & 1
\end{array}\right), \\
& B_{1}=\left(\begin{array}{ll}
1 & 1 \\
0 & 0
\end{array}\right), B_{2}=\left(\begin{array}{ll}
0 & 1 \\
0 & 1
\end{array}\right), \\
& B_{3}=\left(\begin{array}{ll}
0 & 1 \\
1 & 0
\end{array}\right), B_{4}=\left(\begin{array}{ll}
0 & 1 \\
1 & 0
\end{array}\right) .
\end{aligned}
$$

It is easy to check that there exists only one matrix

$$
E=\left(\begin{array}{cccc}
1 & 0 & -\frac{1}{2} & 0 \\
1 & 1 & -\frac{1}{2} & 1 \\
0 & 0 & \frac{1}{2} & 1 \\
0 & 1 & -\frac{1}{2} & 0
\end{array}\right)
$$

such that $\operatorname{res}\left(B_{i}\right)=E \operatorname{res}\left(A_{i}\right)(i=1,2,3,4)$. Since -1 is an eigenvalue of

$$
E^{K}=\left(\begin{array}{cccc}
1 & 0 & 1 & 1 \\
-\frac{1}{2} & 0 & -\frac{1}{2} & 1 \\
0 & 0 & 0 & 1 \\
\frac{1}{2} & 1 & -\frac{1}{2} & 0
\end{array}\right),
$$

$E^{\kappa}$ is not positive semi-definite. Theorem 3.1 implies that there does not exist a quantum channel $\phi$ such that $\phi\left(A_{i}\right)=$ $B_{i}(i=1,2,3,4)$.

Example 2 Let

$$
A_{1}=\left(\begin{array}{ll}
1 & 0 \\
0 & 1
\end{array}\right), A_{2}=\left(\begin{array}{ll}
1 & 2 \\
0 & 3
\end{array}\right),
$$

$$
\begin{aligned}
& A_{3}=\left(\begin{array}{ll}
0 & 1 \\
1 & 0
\end{array}\right), A_{4}=\left(\begin{array}{ll}
0 & 0 \\
0 & 1
\end{array}\right), \\
& B_{1}=\left(\begin{array}{ll}
1 & \frac{1}{2} \\
\frac{1}{2} & 1
\end{array}\right), B_{2}=\left(\begin{array}{ll}
2 & \frac{1}{2} \\
\frac{1}{2} & 2
\end{array}\right), \\
& B_{3}=\left(\begin{array}{ll}
0 & 0 \\
0 & 0
\end{array}\right), B_{4}=\left(\begin{array}{ll}
\frac{1}{2} & 0 \\
0 & \frac{1}{2}
\end{array}\right) .
\end{aligned}
$$

It is easy to check that the only positive semi-definite matrix $E$ satisfying

$$
\operatorname{res}\left(B_{i}\right)=E^{\kappa} \operatorname{res}\left(A_{i}\right)(i=1,2,3,4) \text { and } \operatorname{tr}_{1} E=I_{2}
$$

is

$$
E=\frac{1}{2}\left(\begin{array}{llll}
1 & 0 & 1 & 0 \\
0 & 1 & 0 & 0 \\
1 & 0 & 1 & 0 \\
0 & 0 & 0 & 1
\end{array}\right) .
$$

From Theorem 3.1, we see that there exists a unique quantum channel $\phi$ such that $\phi\left(A_{i}\right)=B_{i}(i=1,2,3,4)$.

A quantum channel $\mathcal{E}$ on $\mathcal{M}_{N}$ is said to be a generalized unitary operation (GUO) if it can be written as

$$
\mathcal{E}(X)=\sum_{j=1}^{d}\left|p_{j}\right|^{2} U_{j} X U_{j}^{\dagger}, \quad \forall X \in \mathcal{M}_{N}
$$

for some $N \times N$ unitary matrices $U_{j}$ and scalars $p_{j}$ with $\sum_{j=1}^{d}\left|p_{j}\right|^{2}=1$.

Clearly, every GUO $\mathcal{E}$ preserves the unit: $\mathcal{E}\left(I_{N}\right)=I_{N}$, equivalently, $\operatorname{tr}_{2}\left(D_{\mathcal{E}}\right)=I_{N}$ (Proposition 2.1(2)).

In Example 2, since $\operatorname{tr}_{2}(E)=\operatorname{tr}_{2}\left(D_{\phi}\right) \neq I_{2}$, the unique quantum channel $\phi$ mapping each $A_{i}$ onto $B_{i}$ is not a GUO. This shows that there does not exist a GUO $\phi$ on $\mathcal{M}_{2}$ sending the given $A_{i}$ to $B_{i}$ for all $i=1,2,3,4$.

Next, let us discuss the existence of generalized unitary operations that send a given family of matrices to another.

Theorem 3.2 Let $\left\{A_{i}\right\}_{i=1}^{k} \subset \mathcal{M}_{N}$ and $\left\{B_{i}\right\}_{i=1}^{k} \subset \mathcal{M}_{N}$. Then there exists a generalized unitary operation $\mathcal{E}$ such that $\mathcal{E}\left(A_{i}\right)=B_{i}(i=1,2, \ldots, k)$ if and only if there exists a group $\left\{\mathbf{a}_{j}\right\}$ of column vectors such that matrices $E_{j}:=\mathbf{a}_{j} \mathbf{a}_{j}^{\dagger}(1 \leqslant$ $j \leqslant d)$ satisfies $\operatorname{tr}_{s} E_{j}=I_{N}(s=1,2)$ and there exists a probability distribution $\left\{q_{j}: j=1,2, \ldots, d\right\}$ such that the matrix $E=\sum_{j=1}^{d} q_{j} E_{j}$ satisfies condition (3.1).

Proof Necessity. Suppose that there exists a generalized unitary operation $\mathcal{E}: X \mapsto \sum_{j}\left|p_{j}\right|^{2} U_{j} X U_{j}^{\dagger}$ such that

$$
B_{i}=\mathcal{E}\left(A_{i}\right)=\sum_{j=1}^{d}\left|p_{j}\right|^{2} U_{j} A_{i} U_{j}^{\dagger}(i=1,2, \ldots, k) .
$$

Put $\mathbf{a}_{j}=\operatorname{res}\left(U_{j}\right)$ and $E_{j}=\mathbf{a}_{j} \mathbf{a}_{j}^{\dagger}$. Then

$$
\operatorname{res}\left(U_{j} \rho U_{j}^{\dagger}\right)=E_{j}^{K} \operatorname{res}(\rho)\left(\forall \rho \in \mathcal{M}_{N}\right), \operatorname{tr}_{s} E_{j}=I_{N}
$$


for all $j$ and $s=1,2$. Let $E=\sum_{j=1}^{d}\left|p_{j}\right|^{2} E_{j}$. Then (3.3) implies that (3.1) holds.

Sufficiency. Suppose that there exists a sequence of matrices $E_{j}=\mathbf{a}_{j} \mathbf{a}_{j}^{\dagger}$ with $\operatorname{tr}_{s} E_{j}=I_{N}(s=1,2)$, where $\mathbf{a}_{j}$ denote some column vectors, such that (3.1) holds for $E=\sum_{j=1}^{d} q_{j} E_{j}$ with $\sum_{j=1}^{d} q_{j}=1$ and $q_{j} \geqslant 0$. Then we reshape the $\mathbf{a}_{j}$ into an $N \times N$ matrix $U_{j}$ such that $\operatorname{res}\left(U_{j}\right)=\mathbf{a}_{j}$ for every $j$. Then it follows from (3.1) that $B_{i}=\sum_{j=1}^{d} q_{j} U_{j} A_{i} U_{j}^{\dagger}$ for $i=1,2, \ldots, k$. Define $p_{j}=\sqrt{q_{j}}$ and

$$
\mathcal{E}(X)=\sum_{j=1}^{d} p_{j}^{2} U_{j} X U_{j}^{\dagger}, \quad \forall X \in \mathcal{M}_{N} .
$$

Then we get a generalized unitary operation $\mathcal{E}$ satisfying $\mathcal{E}\left(A_{i}\right)=B_{i}$ for all $i$.

Example 3 Let

$$
\begin{aligned}
& A_{1}=\left(\begin{array}{ll}
1 & 2 \\
0 & 1
\end{array}\right), A_{2}=\left(\begin{array}{ll}
1 & 3 \\
2 & 0
\end{array}\right), \\
& B_{1}=\left(\begin{array}{ll}
1 & \frac{2}{3} \\
\frac{2}{3} & 1
\end{array}\right), B_{2}=\left(\begin{array}{ll}
\frac{2}{3} & \frac{5}{3} \\
\frac{5}{3} & \frac{1}{3}
\end{array}\right) .
\end{aligned}
$$

It is easy to check that the matrix

$$
\begin{aligned}
& E=\frac{1}{2}\left(\begin{array}{l}
1 \\
0 \\
0 \\
1
\end{array}\right)\left(\begin{array}{llll}
1 & 0 & 0 & 1
\end{array}\right)+\frac{1}{3}\left(\begin{array}{l}
0 \\
1 \\
1 \\
0
\end{array}\right)\left(\begin{array}{llll}
0 & 1 & 1 & 0
\end{array}\right) \\
& +\frac{1}{6}\left(\begin{array}{c}
1 \\
0 \\
0 \\
-1
\end{array}\right)\left(\begin{array}{llll}
1 & 0 & 0 & -1
\end{array}\right) \\
& :=\frac{1}{2} E_{1}+\frac{1}{3} E_{2}+\frac{1}{6} E_{3}
\end{aligned}
$$

satisfies $\operatorname{res}\left(B_{i}\right)=E^{\kappa} \operatorname{res}\left(A_{i}\right)$ for $i=1,2$ and $\operatorname{tr}_{s} E_{j}=I_{2}$ for $j=1,2,3, s=1,2$. Thus, Theorem 3.2 yields that there exists a generalized unitary operation $\phi$ on $\mathcal{M}_{2}$ such that $\phi\left(A_{i}\right)=B_{i}(i=1,2)$. From the proof of Theorem 3.2, we see that the GUO $\phi$ is

$$
\phi: X \mapsto \frac{1}{2} U_{1} X U_{1}^{\dagger}+\frac{1}{3} U_{2} X U_{2}^{\dagger}+\frac{1}{6} U_{3} X U_{3}^{\dagger}(i=1,2),
$$

where

$$
U_{1}=\left(\begin{array}{ll}
1 & 0 \\
0 & 1
\end{array}\right), U_{2}=\left(\begin{array}{ll}
0 & 1 \\
1 & 0
\end{array}\right), U_{3}=\left(\begin{array}{cc}
1 & 0 \\
0 & -1
\end{array}\right),
$$

which are unitary matrices.

\section{An application to duality quantum computers}

Based on the general principle of quantum interference, Long proposed in $[5,6]$ a new type of computing machines called duality quantum computers. Gudder has given the mathematical theory for the duality quantum computer $[9,10]$. For the further discussions and some applications of duality quantum computers, please refer to [7,8,12-14]. In [13], based on Long's idea of duality quantum computers, we proposed complex duality quantum computers (CDQCs) acting on vector-states and operator-states. By [13], a CDQC acting on operator-states is a mapping of the form

$$
\mathbf{C}_{\bar{p}} \circ(\overbrace{i=0}^{n-1} \varepsilon_{i}) \circ \mathbf{D}_{p}: B(\mathcal{H}) \rightarrow B(\mathcal{H})
$$

where $p=\left(p_{0}, p_{1}, \ldots, p_{n-1}\right) \in \mathbb{C}^{n}$ with $\sum_{i=0}^{n-1}\left|p_{i}\right|^{2}=1$, $\left\{\varepsilon_{0}, \varepsilon_{1}, \ldots, \varepsilon_{n-1}\right\}$ a group of CPTP mappings on $B(\mathcal{H})$ and operators

$$
\mathbf{D}_{p}: B(\mathcal{H}) \rightarrow B(\mathcal{H})^{n}, \mathbf{C}_{\bar{p}}: B(\mathcal{H})^{n} \rightarrow B(\mathcal{H})
$$

are given by

$$
\mathbf{D}_{p}(T)=\bigoplus_{i=0}^{n-1} p_{i} T, \mathbf{C}_{\bar{p}}\left(\bigoplus_{i=0}^{n-1} T_{i}\right)=\sum_{i=0}^{n-1} \overline{p_{i}} T_{i} .
$$

Given a CDQC (4.1), we define

$$
\mathbf{U}_{p}=\mathbf{C}_{\bar{p}}\left(\bigoplus_{i=0}^{n-1} \varepsilon_{i}\right) \mathbf{D}_{p}: B(\mathcal{H}) \rightarrow B(\mathcal{H}) .
$$

Then $\mathbf{U}_{p}(T)=\sum_{i=0}^{n-1}\left|p_{i}\right|^{2} \varepsilon_{i}(T)$ for all $T \in B(\mathcal{H})$. That is,

$$
\mathbf{U}_{p}=\sum_{i=0}^{n-1}\left|p_{i}\right|^{2} \varepsilon_{i}
$$

Clearly, $\mathbf{U}_{p}$ is a quantum channel on $B(\mathcal{H})$. Especially, in the case where $\varepsilon_{i}$ is the unitary isomorphism defined by $\varepsilon_{i}(X)=U_{i} X U_{i}^{\dagger}$ for all $i$, the $\mathbf{U}_{p}$ reads

$$
\mathbf{U}_{p}(T)=\sum_{i=0}^{n-1}\left|p_{i}\right|^{2} U_{i} T U_{i}^{\dagger}, \quad \forall T \in B(\mathcal{H})
$$

and therefore is a generalized unitary operation on $B(\mathcal{H})$. Moreover, we say that a CDQC (4.1) is a unitary duality quantum computer (UDQC) if $\varepsilon_{i}$ 's are unitary isomorphisms.

In the case that $\operatorname{dim}(\mathcal{H})=N, B(\mathcal{H})=\mathcal{M}_{N}$. Given input states $\left\{A_{i}\right\}_{i=1}^{k} \subset \mathcal{M}_{N}$ and output states $\left\{B_{i}\right\}_{i=1}^{k} \subset \mathcal{M}_{N}$, Theorem 3.2 gives indeed a necessary and sufficient condition for a UDQC such that $\mathbf{U}_{p}\left(A_{i}\right)=B_{i}(i=1,2, \ldots, k)$ to exist.

This work was supported by the National Natural Science Foundation of China (11171197), the FRF for the Central Universities (GK201104010), and IFGP of SNU (2011CXB004). 
1 Nielsen M A, Chuang I L. Quantum Computation and Quantum information. London: Cambridge University Press, 2000

2 Long G L, Liu Y, Wang C. Allowable generalized quantum gates. Commun Theor Phys, 2009, 51: 65-67

3 Cao H X, Li L, Chen Z L, et al. Restricted allowable generalized quantum gates. Chin Sci Bull, 2010, 55: 2122-2124

4 Zhang Y, Cao H X, Li L. Realization of allowable generalized quantum gates. Sci China-Phys Mech Astron, 2010, 53: 1878-1882

5 Long G L. The general quantum interference principle and the duality computer. Commun Theor Phys, 2006, 45: 825-843

6 Long G L. Mathematical theory of the duality computer in the density matrix formalism. Quantum Inf Process, 2007, 6: 49-53

7 Long G L, Liu Y. Duality computing in quantum computers. Commun Theor Phys, 2008, 50: 1303-1306

8 Long G L. Duality quantum computing and duality quantum information processing. Int J Theor Phys, 2011, 50: 1305-1318

9 Gudder S. Mathematical theory of duality quantum computers. Quantum Inf Process, 2007, 6: 37-48

10 Gudder S. Duality quantum computers and quantum operations. Int J Theor Phys, 2008, 47: 268-279

11 Zou X F, Qiu D W, Wu L H, et al. On mathematical theory of the duality computers. Quantum Inf Process, 2009, 8: 37-50

12 Wang W Y, Shang B, Wang C, et al. Prime factorization in the duality computer. Commun Theor Phys, 2007, 47: 471-472

13 Cao H X, Chen Z L, Guo Z H, et al. Complex duality quantum computers acting on vector-sates and operator-states. Sci China-Phys Mech Astron (in press)
14 Cao H X, Long G L, Guo Z H, et al. Mathematical theory of generalized duality quantum computers acting on vector-states. Int $\mathrm{J}$ Theor Phys, 2012, doi: 10.1007/s10773-012-1225-4

15 Wooters W K, Zurek W H. A single quantum cannot be cloned. Nature, 1982, 299: 802-803

16 Barnum H, Caves C M, Fuchs C A, et al. Noncommuting mixed states cannot be broadcast. Phys Rev Lett, 1996, 76: 2818-2820

17 Luo S L. Using measurement-induced disturbance to characterize correlations as classical or quantum. Phys Rev A, 2008, 77: 022301

18 Luo S L. Quantum discord for two-qubit systems. Phys Rev A, 2008, 77: 042303

19 Wu Y C, Guo G C. Norm-based measurement of quantum correlation. Phys Rev A, 2011, 83: 062301

20 Guo Z H, Cao H X. A classification of correlations of tripartite mixed states. Int J Theor Phys, 2012, doi: 10.1007/s10773-012-1226-3

21 Ferraro A, Aolita L, Cavalcanti D, et al. Almost all quantum states have non-classical correlations. Phys Rev A, 2010, 81: 052318

22 Zhou T, Cui J X, Long G L. Measure of nonclassical correlation in coherence-vector representation. Phys Rev A, 2011, 84: 062105

23 Gisin N. Bell's inequality holds for all non-product states. Phys Lett A, 1991, 154: 201-202

24 Li M, Fei S M, Li-Jost X Q. Bell inequality, separability and entanglement distillation. Chin Sci Bull, 2011, 56: 945-954

25 Choi M D. Completely positive linear maps on complex matrices. Linear Algebra Appl, 1975, 10: 285-290

26 Życzkowski K, Bengtsson I. On duality between quantum maps and quantum states. Open Sys Inform Dyn, 2004, 11: 3-42

Open Access This article is distributed under the terms of the Creative Commons Attribution License which permits any use, distribution, and reproduction in any medium, provided the original author(s) and source are credited. 